\title{
Primary cardiac dedifferentiated liposarcoma in a middle-aged female: a case report
}

\author{
Jiayu Shen ${ }^{1 \dagger}$, Zhi Fang ${ }^{1 \dagger}$, Yahan Zhang ${ }^{2 \dagger}$ and Yingqiang Guo ${ }^{1 *}$
}

\begin{abstract}
Background: Primary malignant cardiac tumors are extremely rare and can present with the same nonspecific characteristics as benign primary cardiac tumors. We herein describe a middle-aged female with an intracavitary, irregular atrial mass who experienced partial surgical resection. The atrial mass which was recognized as myxoma before surgery was finally diagnosed as dedifferentiated liposarcoma (DDLPS) by postoperative pathological examination.

Case presentation: The patient, a 61-year-old female, presented to the emergency room because of progressive chest congestion and shortage of liberties for 6 months and orthopnoea and paroxysmal nocturnal dyspnea for 3 days. The laboratory examinations confirmed no abnormalities. The thoracic computed tomography (CT) scan showed massive hydropericardium, pleural effusion and left atrium occupying lesion. The transesophageal echocardiography (TEE) confirmed an intracavitary and irregular left atrial mass, limiting the mitral valve inflow and pulmonary venous blood reflux. The positron emission tomography/computed tomography (PET/CT) revealed high grade fluorodeoxyglucose uptake only in the intracavitary mass which near the mitral valve. According to operative exploration, the intracavitary mass had invaded the mitral annulus and posterior wall of left ventricle which cannot be resected completely, we did merely partial surgical resection to relieve the patient's symptoms. Postoperative immunohistochemical stain confirmed the diagnosis of DDLPS. The patient was transferred to the oncology department for further therapy. Unfortunately, the patient was detected with brain metastasis 1 month later and died within 5 months after the surgery.

Conclusions: Primary cardiac DDLPS is an extremely rare histological subtype of undifferentiated pleomorphic sarcomas which present the same nonspecific characteristics as benign primary cardiac tumors. Even though surgical resection combined with chemotherapy or radiotherapy remains the mainstream treatment strategy, the prognosis of cardiac malignancy is poor with high mortality. Novel management strategies need to be further explored.
\end{abstract}

Keywords: Dedifferentiated liposarcoma, Heart failure, Intracavitary atrial mass, Pleural effusion, Primary malignant cardiac tumor

\footnotetext{
* Correspondence: 495645314@qq.com

†jiayu Shen, Zhi Fang and Yahan Zhang contributed equally to this work.

'Department of Cardiovascular Surgery, West China Hospital, Sichuan

University, No.37 Guo Xue Alley, Chengdu, Sichuan, People's Republic of

China61004

Full list of author information is available at the end of the article
}

(c) The Author(s). 2019 Open Access This article is distributed under the terms of the Creative Commons Attribution 4.0 International License (http://creativecommons.org/licenses/by/4.0/), which permits unrestricted use, distribution, and reproduction in any medium, provided you give appropriate credit to the original author(s) and the source, provide a link to the Creative Commons license, and indicate if changes were made. The Creative Commons Public Domain Dedication waiver (http://creativecommons.org/publicdomain/zero/1.0/) applies to the data made available in this article, unless otherwise stated. 


\section{Background}

The presence of a heart tumor was first identified in 1559. However, it was not until 1934 that the first clinical diagnosis of a primary heart sarcoma was reported [1]. The autopsy incidence of the primary cardiac neoplasm is extremely low (0.0001-0.030\%) or around 1 in every 500 cardiovascular surgical cases $[2,3]$. The benign cardiac tumors such as myxoma account for $75 \%$. Of the remaining $25 \%$ of tumors that are identified as being malignant, cardiac sarcomas comprise $95 \%$ of cases [4]. The clinical characteristics of the malignant primary cardiac tumors cannot be significantly distinguished from benign primary cardiac tumors. We herein describe a middle-aged female with an intracavitary, irregular atrial mass experienced partial surgical resection. The atrial mass which was suspected as myxoma before surgery was finally diagnosed as DDLPS by postoperative pathological examination.

\section{Case presentation}

A 61-year-old female presented to the emergency room with a 6-month history of progressive chest congestion and shortage of liberties with no abnormal medical history. During the last 3 days, the patient experienced orthopnoea and paroxysmal nocturnal dyspnea. On admission, physical examination revealed body temperature of $37.6{ }^{\circ} \mathrm{C}$, heart rate 120 beats/min, respiratory rate 35 per minute, blood pressure $122 / 75 \mathrm{mmHg}$ and right lung respiratory sound was reduced. Laboratory examinations demonstrated mild leukocytosis (leukocyte count $10.5 \times 10^{9} / \mathrm{L}$ ). Thoracic CT scan revealed occupying lesion in left atrium, accompanied with massive hydropericardium and pleural effusion. (Fig. 1a) Simultaneously right pleural puncture and chest drainage was done to relieve the patient's symptoms. (Fig. 1b) Transesophageal echocardiography (TEE) showed an irregular left atrial mass (measuring $5.0 * 5.2 \mathrm{~cm}$ ), limiting the mitral valve inflow and covering the ostia of the left-side pulmonary veins. (Fig. 1c; Additional file 1) The patient was referred for a PET/CT for further evaluation. Maximum intensity projection images revealed high grade fluorodeoxyglucose uptake only in the intracavitary mass which near the mitral valve. (Fig. 1d).

The patient received emergency surgical intervention under cardiopulmonary bypass since her clinical status deteriorated quickly. The pericardium effusion was drained firstly after pericardiotomy. Operative exploration confirmed complete resection could not be achieved, since the broad intracavitary mass surrounded with fibrotic density had invaded the mitral annulus and posterior wall of left ventricle (Fig. 1e). The intraoperative impression of the frozen section revealed that the lesion was predominantly composed of hyperchromatic spindled cells, and was highly suspicious for spindle cell malignancy. Based on these results, we did merely partial surgical resection only for the protrusive part of the intracavitary mass to minimize the restriction to the pulmonary venous blood reflux and mitral valve flow as much as possible.

After the surgery, the removed left atrium mass was sent for further histopathological examination. Hematoxylineosin staining showed the mass was composed of markedly atypical cells which lack specific morphological features of differentiation (Fig. 2a). Immunohistochemical stain revealed malignant cells strongly positive for MDM2 and CDK4 (Fig. 2b and c), supporting the diagnosis of DDLPS. Postoperative CT scan confirmed the mitral obstruction was totally relieved. (Fig. 2d) The patient was then transferred to the oncology department for further molecular targeting therapy. Unfortunately, the patient was detected with brain metastasis 1 month later (Fig. 2e) and died within 5 months after the surgery.

\section{Discussion and conclusions}

DDLPS is one of the most common types of soft tissue sarcoma which represents over $60 \%$ of all liposarcoma [5] and is almost universally associated with amplification of chromosome segment 12q13-15, which carries the oncogenes MDM2 and CDK4 [6-8]. DDLPS usually originates from adipogenic precursor cells in deep soft tissue, such as that inside the thigh or in the retroperitoneum and frequently presents as a large $(>15 \mathrm{~cm})$, painless, retroperitoneal mass [9]. Being a non-lipotomatous, highly cellular malignancy with a much more aggressive phenotype, DDLPS has a high rate of local recurrence and systemic metastases [10].

In primary cardiac DDLPS, no sex predilection is apparent. Although cases have been identified among a wide range of ages, most patients that are affected are younger than 45 years of age $[11,12]$. The clinical characteristics of the primary cardiac DDLPS mainly depend on the location and infiltration and has no specific characteristics from benign primary cardiac tumors. A study involving 34 patients with primary malignant cardiac tumors observed over a 32-year period reported symptoms such as dyspnoea on exertion (79\%), chest pain $(38 \%)$, cough $(21 \%)$, paroxysmal nocturnal dyspnea (12\%), haemoptysis (12\%), embolic events $(9 \%)$, fever $(9 \%)$, syncope $(6 \%)$, and orthopnoea $(6 \%)[3,13]$. In this case, the intracavitary left atrial mass limited the mitral valve flow and pulmonary venous blood reflux, causing paroxysmal nocturnal dyspnea, orthopnoea and polyserous effusions which are symptoms of left heart failure.

The DDLPS in cardiac chambers often appear as large, irregular, low-attenuation lesions on multidetector CT, 


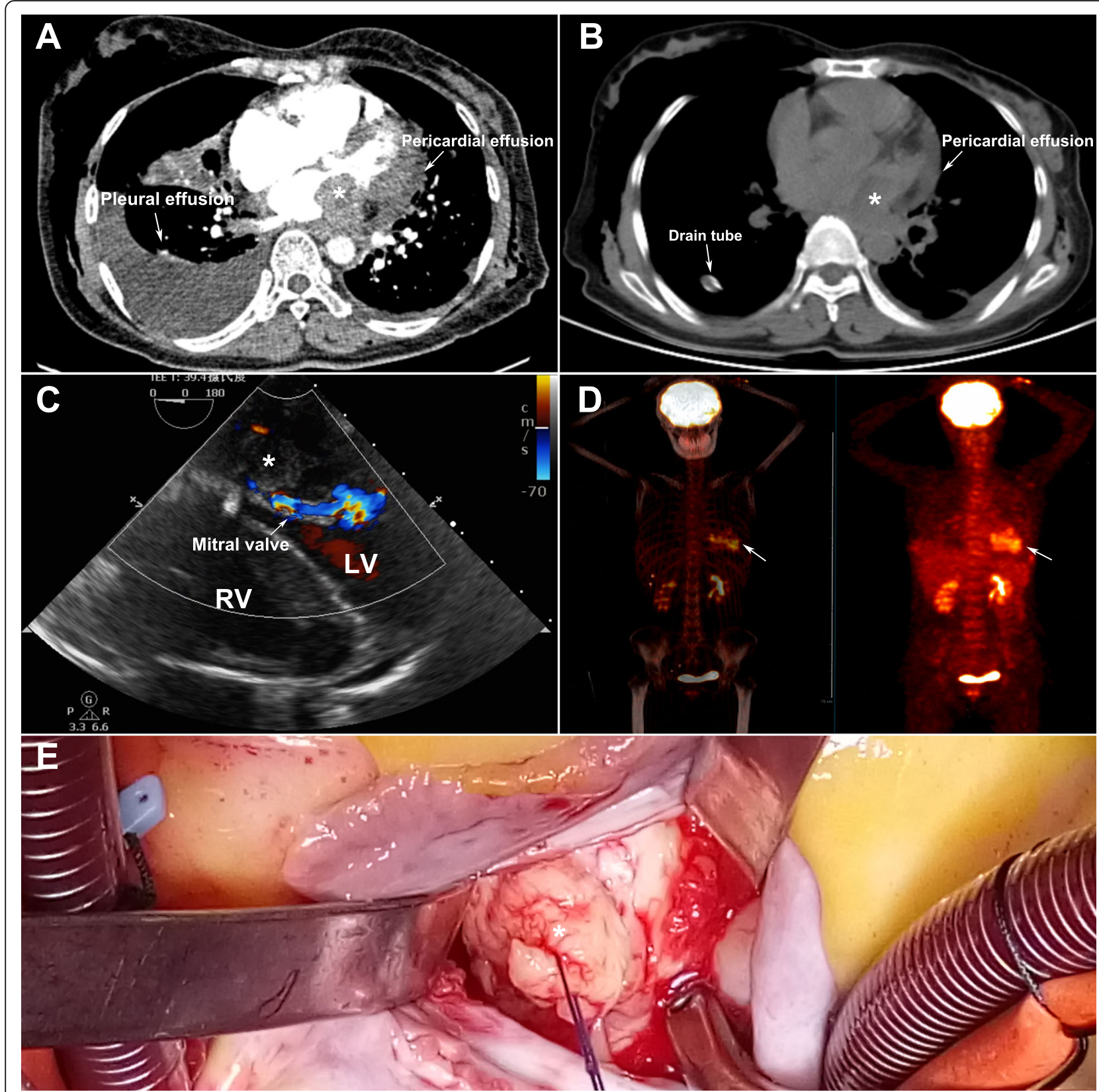

Fig. 1 Preoperative examinations and intraoperative findings: a CT scans revealed occupying lesion in left atrium and massive hydropericardium and pleural effusion (asterisk and arrows); $\mathbf{b}$ The massive right pleural effusion reduced significantly after chest drainage; c TEE showed the left atrial mass limited the mitral valve inflow significantly; d PET/CT confirmed high grade fluorodeoxyglucose uptake only in the intracavitary mass (arrows); e Operative exploration confirmed the atrial mass had invaded the mitral annulus and posterior wall of left ventricle. ${ }^{*}$ The left atrial mass; RV, right ventricle; LV, left ventricle; TEE, Transesophageal echocardiography; PET/CT, Positron emission tomography/computed tomography

and can be either noninvasive/focal or demonstrate infiltration into the myocardium $[14,15]$. As we can observe from the preoperative CT scan, the intracavitary mass has integrated with the myocardium, suggesting the myocardial invasion of the mass. TEE can provide us with more information about the contiguity of the intracavitary mass and other cardiac structures. We also did a further PET/CT for our patient to determine that the intracavitary left atrial mass originates from the myocardium. MRI can also serve as a reference. Like other sarcomas, DDLPS generally appear isointense on T1-weighted images and hyperintense on T2-weighted images, with a heterogenous, delayed-enhancement pattern postcontrast that is related to the underlying composition of the intracavitary mass [16]. Moreover, whole body MRI is a useful technique in detecting distant metastases. However, in this 


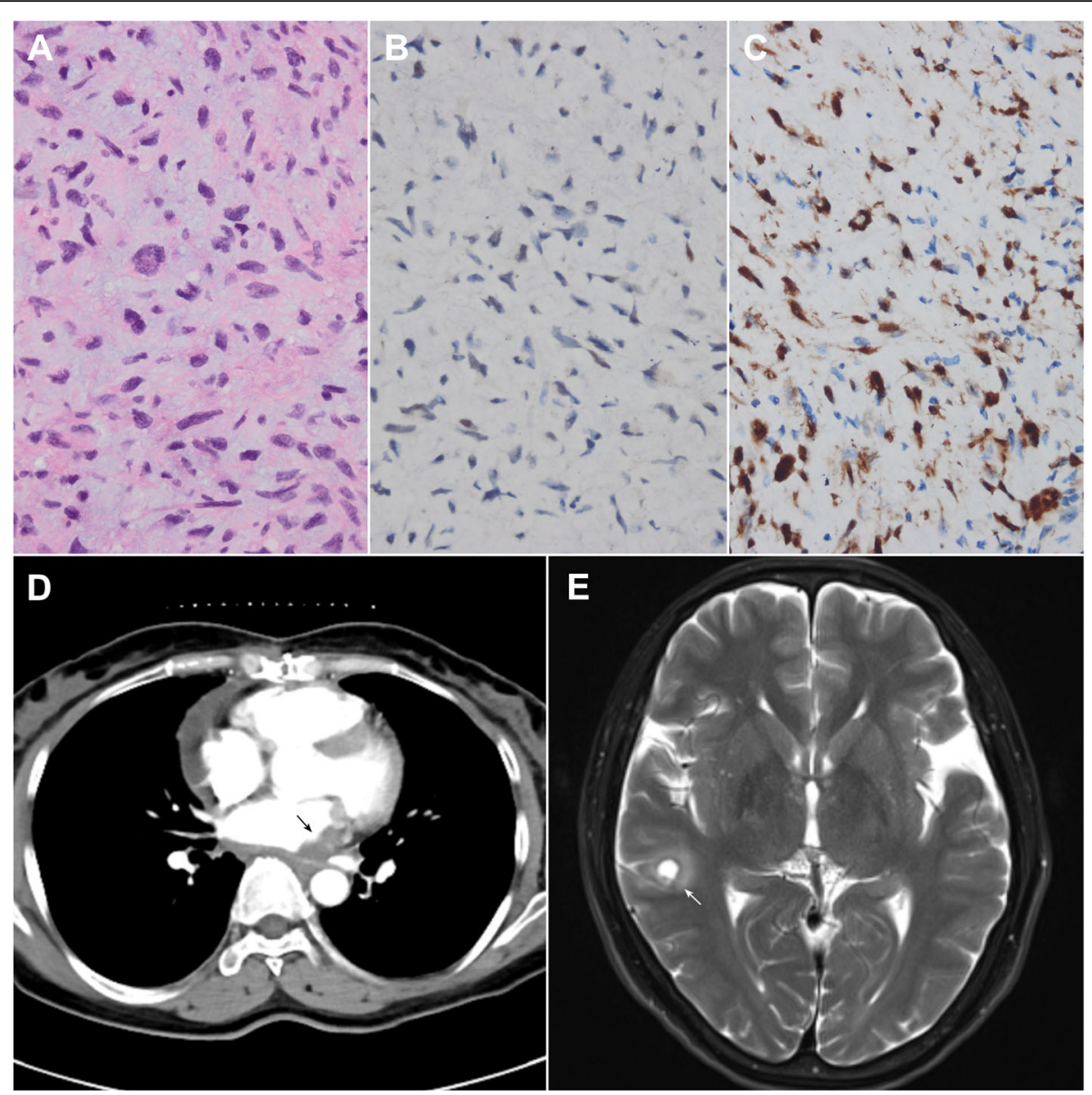

Fig. 2 Pathological findings and follow-up imaging examinations: a Markedly atypical cells which lack specific morphological features of differentiation was found by hematoxylin-eosin staining (H\&E, 400X); $\mathbf{b}$ and $\mathbf{c}$ Immunohistochemical staining of the intracavitary mass is strongly positive for MDM2 and CDK4 (Immunostaining, 400x); d Postoperative thoracic CT scan confirmed the mitral obstruction was totally relieved after partial surgical resection (black arrow); e Postoperative brain MRI examination showed the brain metastasis (white arrow)

case, the deterioration our patient's clinical status seemed to suddenly accelerated after PET-CT and the unstable hemodynamics prevented us from performing other timeconsuming examinations. Unlike angiosarcomas, which seem to have a predilection for the right atrium, primary cardiac DDLPS are mainly left-sided and are not likely to be performed with invasive biopsy. Meanwhile, given that the rarity of primary cardiac DDLPS, data that allow reliable and noninvasive diagnosis are limited and the definitive inferences about optimal therapy remains to be explored.

Even though cardiac tumors present a particular challenge for cardiac surgeons, surgical resection should be attempted in the setting of localized disease, provided the patient has acceptable performance status, in that it seems to provide the best option for palliative care and potential cure $[4,17]$. Complete surgical resection combined with adjuvant chemotherapy and/or local radiotherapy is mandatory to reduce the risk of local recurrence and distant metastasis $[4,13]$. However, the anatomical location and the range of infiltration of the tumor put strain on the acceptance of complete resection as the main treatment modality and it is also worth to mentioning that heart failure is commonly seen in the early course of the left-sided malignant cardiac tumor, and neoadjuvant chemotherapy is usually contraindicated prior to surgery [18]. Implantation of an artificial heart and cardiac transplantation represent an emerging and promising treatment strategy for young patients with no evidence of distant metastasis and isolated unresectable cardiac involvement in recent years [19]. If the hemodynamics of the patient with unresectable tumors remain stable and the donor heart can be obtained in time, heart transplantation can be considered as primary treatment option. However, for patients with initial hemodynamic instability, emergency surgical resection should be done as soon as possible, because this condition can deteriorate in a short time. In this case, as the patient with rapidly deteriorated left heart function, we did emergency partial surgical resection to relieve the restriction on mitral valve flow and 
pulmonary venous blood reflux, leaving the patient in the danger of neoplasm recurrence and metastasis. Even though the patient received regular chemotherapy immediately after surgery, brain metastasis was detected in the early phase and the prognosis was poor.

With a better understanding of the molecular and genetic factors promoting the growth of tumors, more specific and patient-targeted agents such as palbociclib [20] (a cyclin-dependent kinase 4/6 inhibitor used in the treatment of liposarcomas) and olaratumab [21] (a monoclonal antibody directed against platelet-derived growth factor- $\alpha$ for undifferentiated pleomorphic sarcoma and liposarcoma, in combination with doxorubicin) which have been used for certain noncardiac sarcomas may have a role in the treatment of cardiac sarcomas.

In conclusion, primary cardiac DDLPS is an extremely rare histological subtype of undifferentiated pleomorphic sarcomas which present the same nonspecific characteristics as benign primary cardiac tumors. Even though surgical resection combined with chemotherapy or radiotherapy remains the mainstream treatment strategy, the prognosis of cardiac malignancy is still poor with high mortality. Novel management strategies such as molecular targeting therapy in the treatment of cardiac sarcomas needs to be further explored.

\section{Additional file}

Additional file 1: The video of preoperative TEE. (MP4 15574 kb)

\section{Abbreviations}

CT: Computed tomography; DDLPS: Dedifferentiated liposarcoma; PET/ $\mathrm{CT}$ : Positron emission tomography/computed tomography

\section{Acknowledgements}

Not applicable.

\section{Authors' contributions}

All authors participated in the management of the patient in this case report. JYS drafted the manuscript. ZF and YHZ wrote part of discussion and introduction. YQG supervised the case and also supervised the writing of the manuscript. YQG is a chairperson of our department and supervised the entire process. All authors read and approved the manuscript.

\section{Funding}

The postoperative pathological examination was funded by the Health and Family Planning Commission of Sichuan Province (CN) (No.17ZD028).

\section{Availability of data and materials}

All data generated or analyzed during this study are included in this published article.

\section{Ethics approval and consent to participate}

Not applicable.

\section{Consent for publication}

Written informed consent was obtained from the patient for publication of this Case report and any accompanying images. A copy of the written consent is available for review by the editor of this journal.

\section{Competing interests}

The authors declare that they have no competing interests.

\section{Author details}

${ }^{1}$ Department of Cardiovascular Surgery, West China Hospital, Sichuan University, No.37 Guo Xue Alley, Chengdu, Sichuan, People's Republic of China61004. 'Department of Pathology, West China Hospital, Sichuan

University, No.37 Guo Xue Alley, Chengdu 610041, China.

Received: 2 July 2019 Accepted: 13 August 2019

Published online: 30 August 2019

\section{References}

1. Barnes AR, Beaver DC, Snell AMJAHJ. Primary sarcoma of the heart: report of a case with electrocardiographic and pathological studies. American Heart Journal. 1934;9(4):480-91.

2. Look Hong NJ, Pandalai PK, Hornick JL, Shekar PS, Harmon DC, Chen YL, Butrynski JE, Baldini EH, Raut CP. Cardiac angiosarcoma management and outcomes: 20-year single-institution experience. Ann Surg Oncol. 2012;19(8): 2707-15.

3. Ramlawi B, Leja MJ, Abu Saleh WK, Al Jabbari O, Benjamin R, Ravi V, Shapira OM, Blackmon SH, Bruckner BA, Reardon MJ. Surgical treatment of primary cardiac sarcomas: review of a single-institution experience. Ann Thorac Surg. 2016;101(2):698-702.

4. Hoffmeier A, Sindermann JR, Scheld HH, Martens S. Cardiac tumors-diagnosis and surgical treatment. Dtsch Arztebl Int. 2014;111(12):205-11.

5. Conyers R, Young S, Thomas DM. Liposarcoma: molecular genetics and therapeutics. Sarcoma. 2011;2011:483154.

6. Clay MR, Martinez AP, Weiss SW, Edgar MA. MDM2 and CDK4 immunohistochemistry: should it be used in problematic differentiated Lipomatous tumors?: a new perspective. Am J Surg Pathol. 2016;40(12): 1647-52.

7. Barretina J, Taylor BS, Banerji S, Ramos AH, Lagos-Quintana M, Decarolis PL, Shah K, Socci ND, Weir BA, Ho A, et al. Subtype-specific genomic alterations define new targets for soft-tissue sarcoma therapy. Nat Genet. 2010;42(8): 715-21.

8. Dalal KM, Kattan MW, Antonescu CR, Brennan MF, Singer S. Subtype specific prognostic nomogram for patients with primary liposarcoma of the retroperitoneum, extremity, or trunk. Ann Surg. 2006;244(3):381-91.

9. Fletcher CD, Unni KK, Mertens F. Pathology and genetics of tumours of soft tissue and bone, vol. 4. International Agency for Research on Cancer: larc; 2002.

10. Steger CM. Primary liposarcoma of the heart. BMJ Case Rep. 2011;2011: bcr0320114013.

11. Ghadimi MP, Al-Zaid T, Madewell J, Peng T, Colombo C, Hoffman A, Creighton CJ, Zhang Y, Zhang A, Lazar AJ, et al. Diagnosis, management, and outcome of patients with dedifferentiated liposarcoma systemic metastasis. Ann Surg Oncol. 2011;18(13):3762-70.

12. Travis WD, Brambilla E, Burke AP, Marx A, Nicholson AG. Introduction to the 2015 World Health Organization classification of tumors of the lung, pleura, Thymus, and Heart. J Thorac Oncol. 2015;10(9):1240-2.

13. Simpson L, Kumar SK, Okuno SH, Schaff HV, Porrata LF, Buckner JC, Moynihan TJ. Malignant primary cardiac tumors: review of a single institution experience. Cancer. 2008;112(11):2440-6.

14. Anavekar NS, Bonnichsen CR, Foley TA, Morris MF, Martinez MW, Williamson EE, Glockner JF, Miller DV, Breen JF, Araoz PA. Computed tomography of cardiac pseudotumors and neoplasms. Radiol Clin N Am. 2010:48(4):799-816.

15. Araoz PA, Eklund HE, Welch TJ, Breen JF. CT and MR imaging of primary cardiac malignancies. Radiographics. 1999;19(6):1421-34.

16. Motwani M, Kidambi A, Herzog BA, Uddin A, Greenwood JP, Plein S. MR imaging of cardiac tumors and masses: a review of methods and clinical applications. Radiology. 2013;268(1):26-43.

17. Neuville A, Collin F, Bruneval P, Parrens M, Thivolet F, Gomez-Brouchet A, Terrier P, de Montpreville VT, Le Gall F, Hostein I, et al. Intimal sarcoma is the most frequent primary cardiac sarcoma: clinicopathologic and molecular retrospective analysis of 100 primary cardiac sarcomas. Am J Surg Pathol. 2014;38(4):461-9.

18. Blackmon $\mathrm{SH}$, Reardon MJ. Surgical treatment of primary cardiac sarcomas. Tex Heart Inst J. 2009;36(5):451-2. 
19. Agaimy A, Rosch J, Weyand M, Strecker T. Primary and metastatic cardiac sarcomas: a 12-year experience at a German heart center. Int I Clin Exp Pathol. 2012;5(9):928-38.

20. Clark AS, Karasic TB, DeMichele A, Vaughn DJ, O'Hara M, Perini R, Zhang P, Lal P, Feldman M, Gallagher M, et al. Palbociclib (PD0332991)-a selective and potent cyclin-dependent kinase inhibitor: a review of pharmacodynamics and clinical development. JAMA Oncol. 2016;2(2):253-60.

21. van der Graaf WT. Olaratumab in soft-tissue sarcomas. Lancet. 2016; 388(10043):442-4

\section{Publisher's Note}

Springer Nature remains neutral with regard to jurisdictional claims in published maps and institutional affiliations.

Ready to submit your research? Choose BMC and benefit from:

- fast, convenient online submission

- thorough peer review by experienced researchers in your field

- rapid publication on acceptance

- support for research data, including large and complex data types

- gold Open Access which fosters wider collaboration and increased citations

- maximum visibility for your research: over $100 \mathrm{M}$ website views per year

At BMC, research is always in progress.

Learn more biomedcentral.com/submissions 